\title{
Stable Symplectic Integrators for Power Systems
}

\author{
Daniel Okunbor and Emmanuel Akinjide \\ Department of Mathematics and Computer Science, \\ University of Maryland Eastern Shore \\ Princess Anne, Maryland 21853
}

\begin{abstract}
The paper illustrates the application of symplectic integrators obtained by composition for solving power system consisting of several machines. The multi-machine angular swings during and following fault conditions and clearing are investigated. Numerical results obtained using symplectic integrators were found to be comparable to those obtained using the traditional trapezoid integrator. Symplectic integrators give globally stable results and save computational time, necessary for multi-machine power systems.
\end{abstract}

Keywords: Hamiltonian systems, swing equations, symplectic integrators, power systems, Lie group.

\section{Introduction}

There has been significant research in the computer solution of dynamic Hamiltonian system generally defined by scalar-valued, sufficiently smooth Hamiltonian function $H(z)$ on an even-dimenstional space, $z \in \mathcal{R}^{2 d}$, see $[2,7,11]$. The correspending Hamiltonian system of ordinary differential equations is

$$
\frac{d}{d t} z=J \nabla H(z)
$$

where $z=\left(\begin{array}{c}q \\ p\end{array}\right) \in \mathcal{R}^{2 d}, J=\left(\begin{array}{cc}O_{d} & I_{d} \\ -I_{d} & O_{d}\end{array}\right), I_{d}$ is an identity matrix of dimension $d$. The increased in research interest in Hamiltonian system has been due largely to its applications in science and engineering. Two applications in which dynamical Hamiltonian system has been used extentively are bimolecular modeling and $N$-body simulation of planetary system $[8$, 9]. The general form of separable Hamiltonian for such applications is,

$$
H(q(t), p(t))=T(p)+V(q)=\frac{1}{2} p^{T} M^{-1} p+V(q)
$$


where $q(t), p(t) \in \mathcal{R}^{d}$ are respectively, the positions and momenta of the system and $M$ is the diagonal mass matrix. The corresponding Hamiltonian system is

$$
\frac{d q}{d t}=M^{-1} p, \frac{d p}{d t}=-\nabla V(q),
$$

where $-\nabla V(q)$ is the force of the system.

Symplectic integrators $[7-9,11]$ have been developed in favor of the conventional numerical solution techniques for Hamiltonian system of ordinary differential equations. Symplectic integrators preserve the Poincaré integrals of the $t$-flow of the Hamiltonian system except for truncation error. Comparison of symplectic integrators with conventional non-symplectic integrator revealed the stable characteristics of symplectic integrators making them appropriate for long-time integration.

In this paper, we investigate the application of Hamiltonian system to power system consisting of $m$ machines. With reasonable assumption on the asychronous speed, the swing equations are modeled as a Hamiltonian system, allowing for the application of symplectic integrators. The numerical results indicate a very strong promise for symplectic integrators for power systems. The symplectic integrators tested were approximately ten times computationally faster than the conventional trapezoid-based method commonly used in power systems. The stable nature of the solution obtained utilizing symplectic integrators make them suitable for transient stability analysis as demonstrated in the paper. Section 2 describes symplectic integration schemes. The Lie group approach is used to construct symplectic integrators via composition of lower-order integrators. Section 3 focuses on the derivation of the swing equations of power systems and their relationship to the separable Hamiltonian in eq (2). This section also addresses numerical experients using a power system consisting of three machines and nine buses.

\section{$2 \quad$ Symplectic Integration}

Let $z\left(t_{1}\right)$ be the solution of Hamiltonian system (1) at time $t_{1}$ and $z\left(t_{2}\right)$, the solution at time $t_{2}$, where $t_{2}>t_{1}$, we write

$$
z\left(t_{2}\right)=\Psi_{(t, H)}\left(z\left(t_{1}\right)\right),
$$

where $\Psi_{t}$ is the transformation function from $z\left(t_{1}\right)$ to $z\left(t_{2}\right)$. A symplectic transformation is one satisfying

$$
\left(\frac{\partial \Psi_{t}}{\partial z}\right)^{\mathrm{T}} J\left(\frac{\partial \Psi_{t}}{\partial z}\right)=J
$$


A symplectic integrator $z_{n+1}=\Psi_{(h, H)}\left(z_{n}\right)$, where $z_{n+1}$ is the numerical solution at $t=t_{n+1}$ and $z_{n}$ is the solution at $t=t_{n}$, is one for which $\Psi_{h, H}$ is symplectic, see $[7,11]$. There are several approaches for constructing symplectic integrators $[8,11]$, we would use the Lie group approach. The Lie group approach involves the composition of lower-order integrators to obtain higher-order integrator. Let the time derivatives of $\left.z(t)=(q(t))^{\mathrm{T}}, p(t)^{\mathrm{T}}\right)^{\mathrm{T}} \in \mathcal{R}^{2 d}$ be expressed as

$$
\frac{d^{k}}{d t^{k}} z(t)=D_{H}^{k} z(t)
$$

for the differential operator $D_{H}$ defined by $D_{H}=\sum_{i=1}^{d}\left(\frac{\partial H}{\partial p_{i}} \frac{\partial}{\partial q_{i}}-\frac{\partial H}{\partial q_{i}} \frac{\partial}{\partial p_{i}}\right)$. Since

$$
z(t)=\sum_{k=0}^{\infty} \frac{t^{k}}{k !} \frac{d^{k}}{d t^{k}} z(0)=e^{t D_{H}}(z(0))
$$

the exponential operator maps $z(0)$ to $z(t)$. For separable Hamiltonian, the differential operator is expressed as $D_{H}=D_{T}+D_{V}$, and the exponential operator (5) applied to some $z=\left(q^{\mathrm{T}}, p^{\mathrm{T}}\right)^{\mathrm{T}}$ can be approximated by a systematic application of following operators.

$$
e^{t D_{T}}(z) \equiv\left(\begin{array}{c}
q+t \nabla T(p) \\
p
\end{array}\right), e^{t D_{V}}(z) \equiv\left(\begin{array}{c}
q \\
p-t \nabla V(q)
\end{array}\right) .
$$

These transformations are both symplectic since they satisfy the necessary and sufficient condition for a mapping to be symplectic. It is easy to show that the composition of symplectic transformations is also symplectic. A single step second-order symplectic integrator can be expressed as the composition of first-order symplectic integrators namely,

$$
z_{n+1}=\Psi_{h, H}^{[2]}\left(z_{n}\right)=e^{\frac{h}{2} D_{V}} e^{h D_{T}} e^{\frac{h}{2} D_{V}}\left(z_{n}\right) .
$$

Using the Baker-Campbell-Hausdorff $(\mathrm{BCH})$ formula from Lie algebra $[2,7]$, the product of exponentials of noncommuntative operators can be expressed as

$$
e^{A} e^{B}=e^{A+B+\frac{1}{2}[A, B]+\frac{1}{12}([[B, A], A]+[B,[B, A]])-\frac{1}{24}[B,[[B, A], A]]+\cdots,}
$$

where we have adopted the Poisson bracket notation for noncomutative operators, viz: $[A, B]=A B-B A$. Clearly, the composition method is second-order accurate, since its operator approximates $e^{h D_{H}}$ up to the $h^{3}$ term:

$$
e^{\frac{h}{2} D_{V}} e^{h D_{T}} e^{\frac{h}{2} D_{V}}=e^{h D_{H}+\frac{h^{3}}{24}\left(2\left[D_{T},\left[D_{T}, D_{V}\right]\right]-\left[\left[D_{T}, D_{V}\right] D_{V}\right]\right)+0\left(h^{5}\right)}
$$


The composition of $\Psi_{h, H}^{[2]}$ will give a fourth-order symplectic integrator:

$$
\Psi_{h, H}^{[4]}=\Psi_{x_{1} h, H}^{[2]} \Psi_{x_{0} h, H}^{[2]} \Psi_{x_{1} h, H}^{[2]} .
$$

Yoshida [11] derived a 4-th order with the following parameters

$$
x_{1}=\frac{1}{3}\left(2+2^{\frac{1}{3}}+2^{-\frac{1}{3}}\right), x_{0}=1-2 x_{1},
$$

using Lie Algebra.

\section{Application of Hamiltonian System to Power Systems}

In this section, we would describe the equation governing the motion of the rotors in power system consisting of synchronous $m$ generators and buses. The equation is based on the principle of dynamics that states that accelerating torques is the product of the moment of inertia of the $k$-th rotor and its angular acceleration, see $[1,3,6,10,12,13]$.

$$
G \alpha_{k}(t)=f_{k}(t)-f_{e}(t)=f_{a}(t)
$$

where $G$ is the total moment of inertia of the rotating masses in $\mathrm{kgm}^{2}$, $\alpha_{k}$ is the $k$-th rotor angular acceleration in $\mathrm{rad} / \mathrm{sec}^{2}, f_{k}$ is the mechanical torque supplied by the prime mover minus the retarding torque due to mechanical losses in $N m, f_{e}$ is the electrical torque that account for the total 3-phase electrical power output of the generator including the electrical losses in $N m$, and $f_{a}$ is the net accelerating torque in $N m$. The rotor angular acceleration is given by

$$
\begin{aligned}
& \alpha_{k}(t)=\frac{d \omega_{k}(t)}{d t}=\frac{d^{2} \theta_{k}(t)}{d t^{2}}, \\
& \omega_{k}(t)=\frac{d \theta_{k}(t)}{d t}
\end{aligned}
$$

where $\omega_{k}$ is the rotor angular velocity in $\mathrm{rad} / \mathrm{sec}$, and $\theta_{k}$ is the rotor angular postion with respect to a synchronous reference axis in radians. In steady state, $f_{k}$ and $f_{e}$ are equal, and the accelerating torque $f_{a}$ is zero due to the synchronous speed (constant velocity). $f_{a}$ and $\alpha_{k}$ are positive with an increasing rotor speed, when $f_{k}$ greater than $f_{e}$. A decreasing rotor speed is obtained when $f_{k}$ is less than $f_{e}$. It is easier to measure rotor angular position with respect to synchronously rotating reference. 
The above equations (4) are combined to form the power generated from a typical generator. Let

$$
\theta_{k}(t)=\omega_{\mathrm{syn}}(t)+\delta_{k}(t)
$$

where $\omega_{\text {syn }}$ is the synchronous angular velocity in $\mathrm{rad} / \mathrm{sec}, \delta_{k}$ is the rotor angular positon with respect to a synchronously rotating reference in radians and

$$
G \frac{d^{2} \theta_{k}(t)}{d t^{2}}=G \frac{d^{2} \delta_{k}(t)}{d t^{2}}=f_{k}(t)-f_{e}(t)=f_{a}(t) .
$$

Multiplying the above equation by $\omega_{\text {syn }}(t)$ and dividing by its rated mega voltage amperes $\left(S_{\text {mach }}\right)$ gives the per-unit asychronous speed. This is easier to work with in electrical power system and it is this case that gives rise to separable Hamiltonian. Let $H$ represents the energy of system normalized by a rated mega voltage amperes $\left(S_{\text {mach }}\right)$

$$
H=\frac{\text { Stored kinetic energy in megajoules at synchronous speed }}{\text { Machine rating in mega voltage amperes }} .
$$

In terms of the moment of inertia, $H=\frac{\frac{1}{2} G \omega_{\mathrm{syn}}}{S_{\mathrm{mach}}}$, where $G=\frac{2 H}{\omega_{\mathrm{syn}}} S_{\text {mach }}$. The resulting swing equations are

$$
\frac{2 H}{\omega_{\text {syn }}} \frac{d^{2} \delta_{k}}{d t^{2}}=\frac{f_{a}}{S_{\text {mach }}}=\frac{f_{k}-f_{e}}{S_{\text {mach }}},
$$

for $k=1,2, \ldots, m$. This fundamental system of ordinary differential equations governs the rotational dynamics of the synchronous machine in most power systems. In Figure 1, the angle between E (the generator voltage) and $\mathrm{V}$ (output voltage) is called phase angle. The phase angles of the internal voltages depend on the positions of the rotors of the machines. If synchronous speed were not maintained between the generators of a power system, the phase angles of their internal voltages would be changing constantly. This will be in relation to the performance of each machine. Invariably, as a result this, a satisfactory operation would be impossible. The phase angles and the internal voltages of synchronous machines will remain constant only if the speeds of the other machines remain constant. This can happen only at the speed corresponding to the frequency of the reference phasor. If there is a change in the load profile of the entire system or in any of the generators, the current in that particular generator or the entire generator's will change to meet the demand. If the current does not effect any change, the phase angles of the internal voltages will 

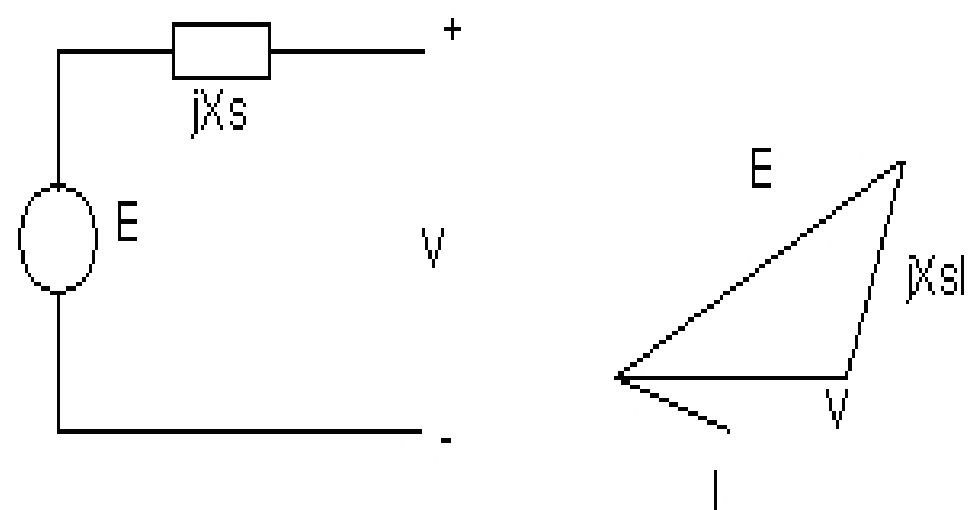

Fig. 1. Angle Diagrams

not change. There will be momentary changes in speed to demand the adjustment to the angles of the voltages to each machine. This is because the phase angles are determined by the relative positions of the rotors. This change is what is called disturbance in electrical system. When the machines have adjusted to the new positions mentioned or the cause of the change has been removed, the machines must operate at synchronous speed. If one machine does not return to synchronous state with the rest of the machines, large currents will circulate.

Stability studies are classified according to whether they involve steady state or transient conditions. An ac generator cannot deliver more than the rated power nor can synchronous motor absorb more than rated limit. Disturbances on a system that are caused by suddenly applied loads, or by the occurrence of faults, loss of excitation in the field of a generator, or switching, may cause loss of synchronous when the change in the system by the disturbance would not exceed the stability limit if the change were made gradually. The limiting value of power is called the transient stability or the steady-state stability limit, according to whether a sudden or gradual change in conditions reaches the point of instability of the system.

\section{Three Machine, Nine-bus Power System}

The experiments are based on a nine-bus and three-generator power system (see [5]), connected with transformers and transmission lines and associated relays as depicted in Figure 2. Transmission lines are classified 


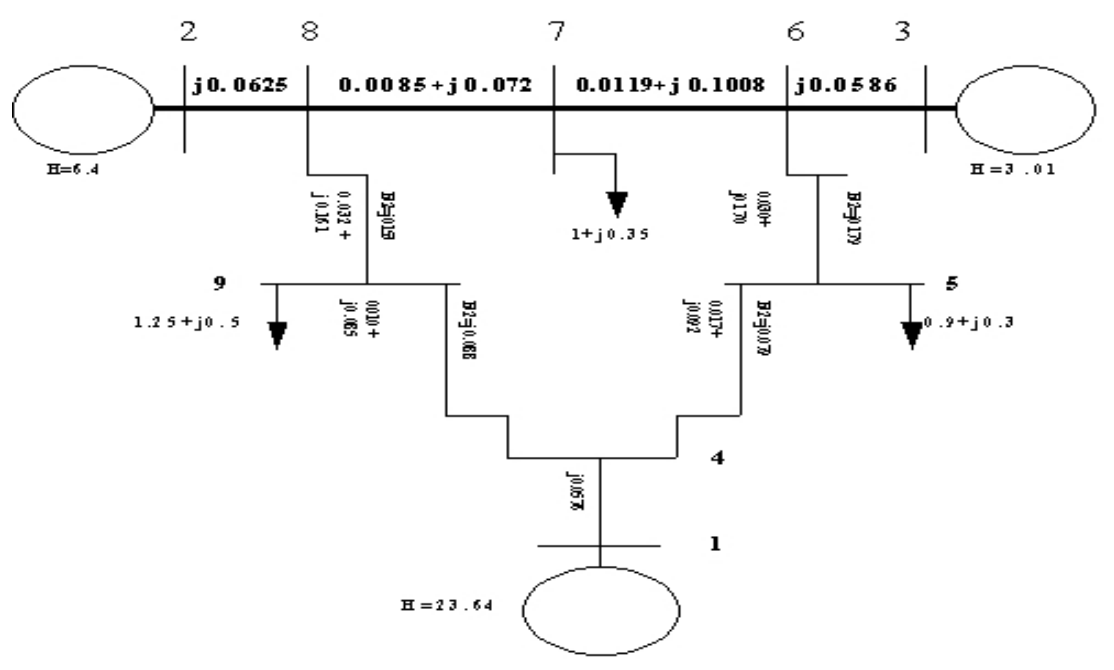

Fig. 2. Three-Machine, Nine-Bus Power System

as either single phase or three phase system. They are 60- Hz synchronous generated. They are connected and transmitted in Wye or Delta form. Usually, the transmission lines are connected to infinite bus meaning that there is no variation in the load voltage with the load voltage as a reference and at zero degrees angle. In the generating plants, paralleling of generators is necessary to supply the loads for occasional critical or peak loads. They are grouped in three to supply one-third load and for spare capacity. To parallel three generators, the output voltages of each must have the same magnitude, frequency, phase rotation, and phase angle. The same phase rotation means that the voltages of the corresponding phases of each generator must come to their peaks at the same time. In this experiment, the three generators are connected either in Wye or Delta form. The lines are represented as a single one-line diagram. In reality, there are three conductors for a three phase and two conductors for a single phase systems. The maximum power a generator can tranfer is indicated in Figure 1 and by this equation.

$$
p=\frac{|E||V|}{X} \sin \delta
$$

The power transferred also depend on the variation of the angle and the generator's speed. The experiments are based on three phase faults on the 
transmission lines. It is also assumed that there is a three phase faults on line 1 and line 2 . The moment of inertia, angular deviation, and velocity for the experiments are shown in Figure 2. The results of the experiment indicate symplectic response time are faster in CPU time than the trapezoid integration methods. A second-order simulation was performed using symplectic integration with clearing time of 0.192 seconds. Figures 3 and 4 indicate faster response time. We also extended this experiment to fourth-order using symplectic integrator. The outcomes of the experiments are displayed in Figures 5 and 6.
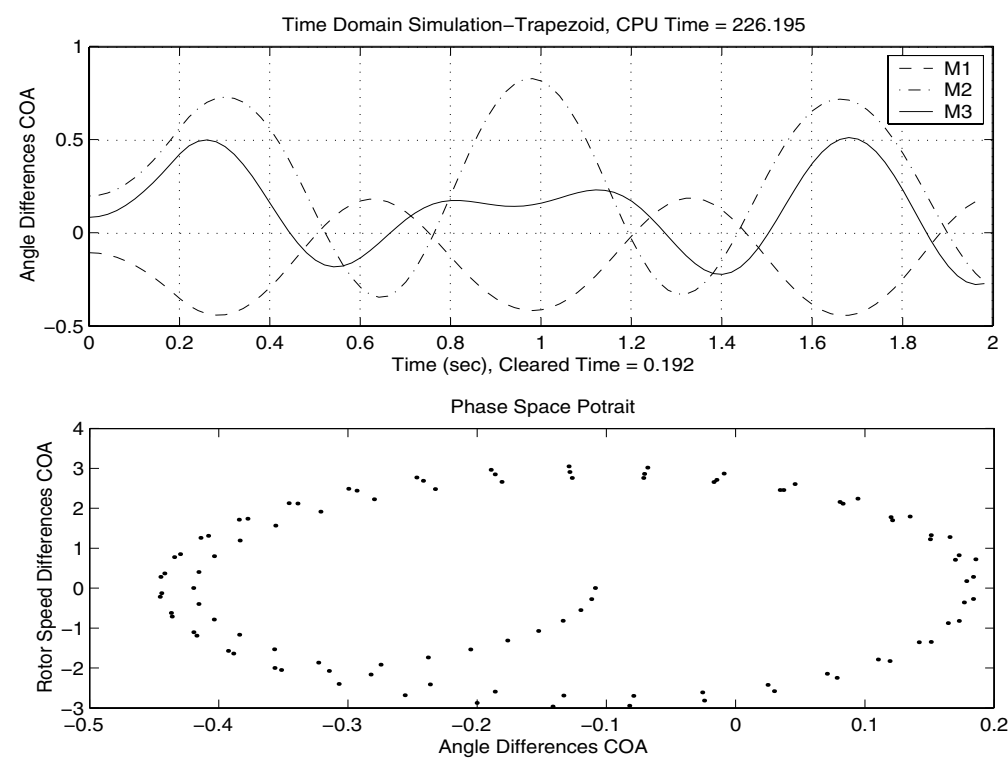

Fig. 3. Phase Angles Using Second-Order Non-Symplectic Integrator

\section{References}

1. R. Ando and S. Iwamoto, "Highly reliable transient solution method using energy function (power systems)", Electrical Engineering in Japan, vol 108, no.4, p.57-66, 1988.

2. V. I. Anold, Mathematical Methods of Classical Mechanics, Springer-Verlag, 1989.

3. J. H. Bentley, A programmed Review for Electrical Engineering, Engineering Press, Inc.,1994.

4. H. D. Chiang, B. Y. Ku, and J. S. Thorp, "A constructive method for direct analysis of transient stability", IEEE, p.3 vol.2508, 684-689, vol.1,1988.

5. K. R. Folken, The Transient Energy Function Method and Thee-Phase Unbalanced Systems, Master's Thesis, University of Missouri-Rolla, 1997. 

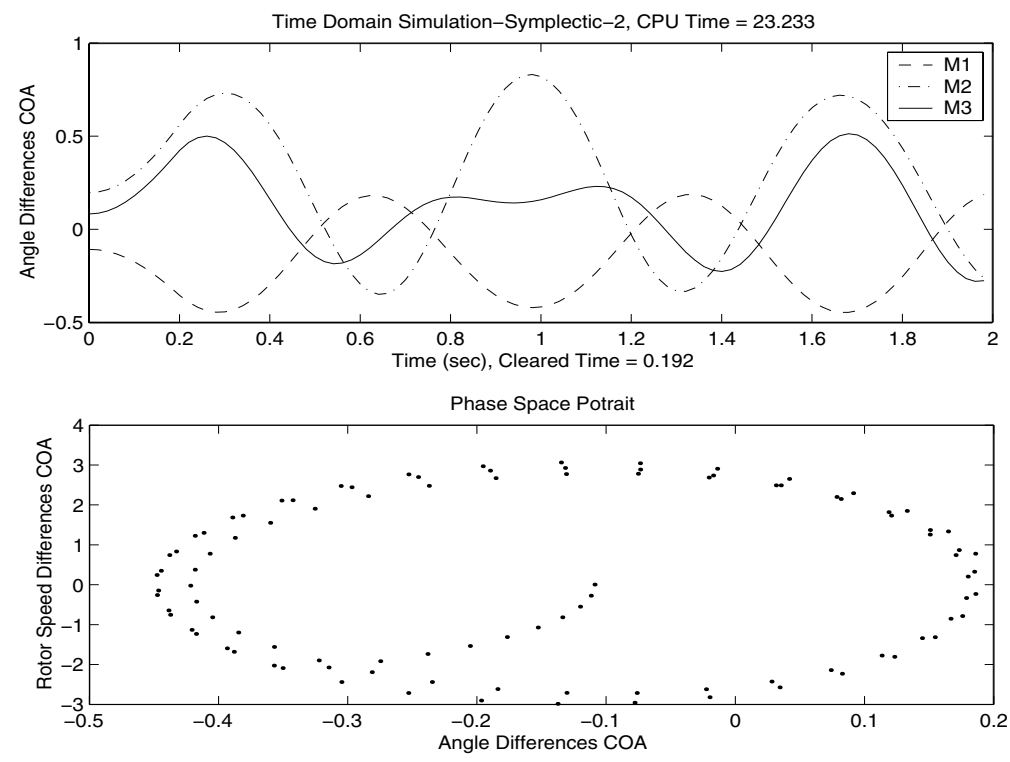

Fig. 4. Phase Angles Using Second-Order Symplectic Integrator

6. A. A. Fouad, et. al., "Direct transient assessment with excitation control", IEEE Transactions on power systems, vol.4, no.1, p.75-82, 1988.

7. E. Hairer, S. P. Norsett, and G. Wanner, Solving Ordinary Differential Equations I: Nonstiff Problems, Springer-Verlag, 1991.

8. D. Okunbor and R. D. Skeel, "Explicit Canonical Methods for Hamiltonian Systems", Math. Comp. 59(200), p.439-455, 1992.

9. D. Okunbor and D. Hardy, "Symplectic Multiple Time Step Integration of Hamiltonian Dynamical Systems", Procs. Dynamic Systems and Applications, vol 3, p.48390, 2001.

10. M. A. Pai, P. W. Sauer, and F. Dobraca, "A new approach to transient stability evaluation in power systems", IEEE, vol 1, 676-780, 1988.

11. J. M. Sanz-Serna and M. P. Calvo, Numerical Hamiltonian Problems, Chapman and Hall, 1994.

12. W. D. Stevenson, Jr., Elements of Power system analysis. McGraw Hill, 1982.

13. A. Seidman, H. W. Beaty, and H. Mahrous, Handbook of Electric Power Calculations, McGraw Hill 1984. 

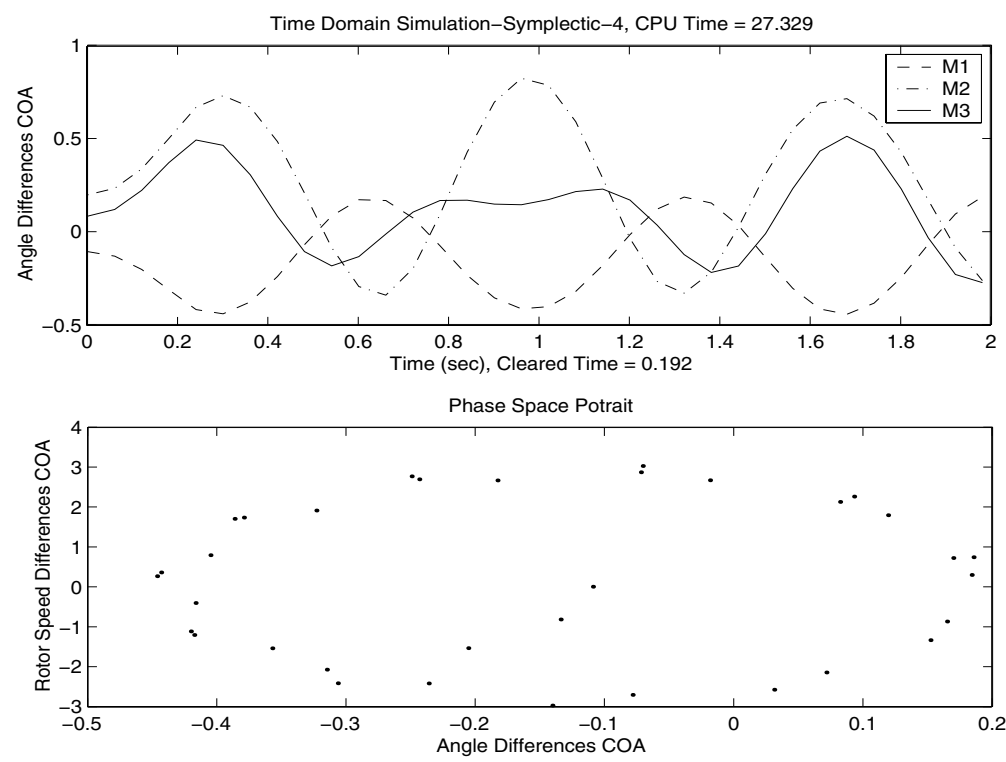

Fig. 5. Phase Angles Using Fourth-Order Symplectic Integrator
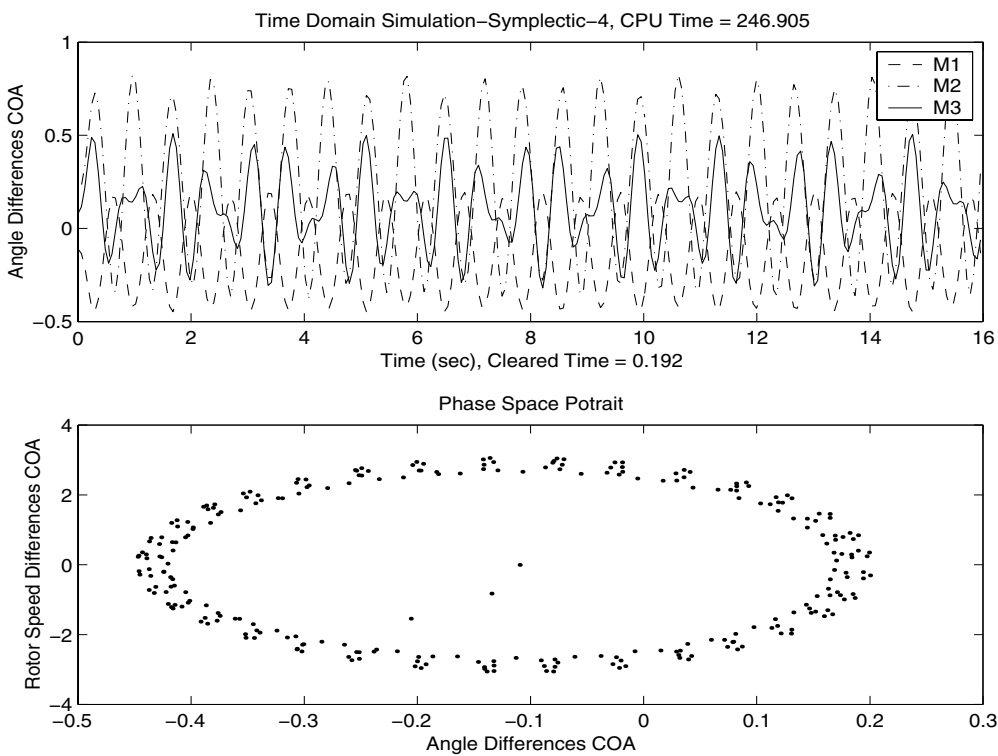

Fig. 6. Extended Time Fourth-Order Symplectic Integration Results 